\title{
Ion Source for Neutral Beam Injection Meant for Plasma and Magnetic Field Diagnostics ${ }^{\mathrm{a}}$
}

\author{
Jaakko Hannes Vainionpaa, Ka Ngo Leung, Richard A Gough, and Joe W Kwan \\ Lawrence Berkeley National Laboratory, Berkeley, CA, USA
}

\author{
Fred Levinton
}

NOVA Photonics Inc., Princeton, NJ, USA

\begin{abstract}
At the Lawrence Berkeley National Laboratory (LBNL) a diagnostic neutral beam injection system for measuring plasma parameters, flow velocity, and local magnetic field is being developed. The systems is designed to have a $90 \%$ proton fraction and small divergence with beam current at 5-6 A and a pulse length of $\sim 1 \mathrm{~s}$ occurring once every 1-2 min. The ion source needs to generate uniform plasma over a large $(8 \mathrm{~cm} \times 5$ $\mathrm{cm})$ extraction area. For this application, we have compared RF driven multicusp ion sources operating with either an external or an internal antenna in similar ion source geometry. The ion beam will be made of an array of six sheet-shaped beamlets. The design is optimized using computer simulation programs.
\end{abstract}

\section{INTRODUCTION}

\footnotetext{
${ }^{a}$ Contributed paper, published as part of the Proceedings of the 12th International Conference on Ion Sources, Jeju, Korea, August 2007.
} 
At the Lawrence Berkeley National Laboratory (LBNL) a diagnostic neutral beam injection system is being developed in collaboration with Nova Photonics Inc. The neutral beam can be used to measure the ion temperature ${ }^{12}$ and flow velocity ${ }^{3}$, plasma fluctuations ${ }^{4}$ and local magnetic fields inside the plasma ${ }^{5}$. High proton fraction and small divergence are essential for diagnostic neutral beams. In our design, a neutral hydrogen beam with an $\mathrm{x}=8 \mathrm{~cm}$ and $\mathrm{y}=11 \mathrm{~cm}$ or smaller elliptical beam spot at $2.5 \mathrm{~m}$ from the end of the extraction column is produced. This corresponds to a full angular divergence of $<60 \mathrm{mrad}$ in the $\mathrm{y}$ direction and $<10 \mathrm{mrad}$ in the $\mathrm{x}$ direction. The beam will deliver a beam current of 5-6 A with $\sim 35 \mathrm{keV}$ energy to the target with a pulse width of $\sim 1 \mathrm{~s}$, once every $1-2$ min. The $\mathrm{H}_{1}{ }^{+}$ion fraction of the hydrogen beam will exceed $90 \%$. The ion source needs to generate uniform plasma over a large $(8 \mathrm{~cm} \times 5 \mathrm{~cm})$ extraction area.

The ion beam will be extracted and accelerated by a set of grids with slits, thus forming an array of 6 sheet-shaped beamlets. The multiple grid extraction will be optimized using computer simulation programs. Neutralization of the beam will be done in a gas cell, which is expected to have $\sim 70 \%$ neutralization efficiency. A schematic diagram of the neutral beam injection system is shown in Fig. 1. In this article we describe the ion source and the extraction design.

For this application, we have compared two types of RF driven multicusp ion sources operating at $13.56 \mathrm{MHz}$. The first one is an ion source with an external spiral antenna behind a dielectric RF-window. The second one uses an internal antenna in a similar ion source geometry. 


\section{ION SOURCE}

With a $6 \sim 8 \mathrm{~cm}$ long slit extraction, the required current density is $150-250 \mathrm{~mA} / \mathrm{cm}^{2}$. According to ion trajectory simulations, the plasma uniformity over the extraction area needs to be within $10 \%$. We have tested ion sources with either internal or external RFantenna as shown in Fig. 2. The RF-power is impedance matched to the ion source by using a step down matching network ${ }^{6}$.

The ion source is built with an aluminum body and has multicusp magnetic field to confine the plasma and improve the plasma uniformity. The external antenna is spiral shaped and is made of water-cooled copper tubing. The back flange/RF-window is a movable quartz disk that allows adjustment of the source length. The internal antenna, which has two turns, is constructed by inserting a wire inside a water-cooled quartz tube. In this case the back flange is an aluminum disk with multicusp magnets.

Low operation pressure is important because it reduces charge exchange and voltage breakdown problems in the extraction region. As shown in Fig. 3 the sources operated effectively under 8 mTorr pressure. Especially the source with the internal antenna operated at low pressures having optimum operating pressure $\sim 5 \mathrm{mTorr}$. The impurities were found to be high during a recent tests resulting in an effective ion mass of $\sim 6 \mathrm{amu}$.

Nevertheless previous experiments ${ }^{78}$ at LBNL with similar sources have found that the 
effective mass was $\sim 1.2 \mathrm{amu}$. We suspected that the problem was due to impurities from the aluminum wall and are planning to change the wall material to alumina.

For RF ion sources the extracted ion current vs. RF power behaves quite linearly. There are commercially available $13.56 \mathrm{MHz}$ RF-power sources up to $15 \mathrm{~kW}$. In Fig 4 we can estimate that both sources can deliver the required current densities with $<15 \mathrm{~kW}$ of $\mathrm{RF}$ power.

The aspect ratio of (source depth)/(source diameter) affect both extracted current density and plasma uniformity. For external antenna the aspect ratio was $1 / 4$. For the internal antenna the aspect ratio was $1 / 2$. Small aspect ratio enables the external antenna to generate higher plasma densities (higher power density) in Fig. 4 with less ideal plasma uniformity in Fig. 5. In Fig. 5 the source with internal antenna is capable of producing the required plasma uniformity $(<10 \%)$. However, with the external antenna, the uniformity is only marginally acceptable. Nevertheless, we believe that by increasing the aspect ratio in the external antenna source we can improve the plasma uniformity.

\section{EXTRACTION}

Ion source extraction is designed by using particle trajectory simulation code IBsimu ${ }^{10}$ that can handle multiple beamlets (including the electrostatic interaction between 
beamlets). For single slit problem the newly developed IBsimu was benchmarked against the more established BPGUNS ${ }^{11}$. The beam has strict divergence $(<10 \mathrm{rad})$ and beam spot size $(<8 \mathrm{~cm})$ requirements in the $\mathrm{x}$ direction.

We have considered both multiple-slit and multiple-hole extraction. The multiple-slit extraction (see Fig 6) was selected due to the very small divergence in the long direction (x) of the slit and high transparency of the slit design. The beam is extracted from 6 slits, $\sim 8 \mathrm{~cm}$ long, using simple triode extraction. The length of the extraction section needs to be minimized in order to maximize vacuum pumping and minimize the heating of the electrodes caused by charge exchange in the extraction region. The specially shaped plasma electrode produces focusing fields that are very linear and thus the aberrations in the beam emittance are smaller.

Due to the large extraction area, the pressure in the extraction gap is significant. This design keeps the voltage gradients at $\sim 50 \mathrm{kV} / \mathrm{cm}$ to prevent sparking problems. For such a long drift distance $(\sim 2.5 \mathrm{~m})$ to the target the overall machining/alignment tolerances need to be less than 20 micron in the y direction. As shown in Fig. 7 the design can deliver the beam well inside the required divergence $<60 \mathrm{mrad}$ and beam spot of $<11 \mathrm{~cm}$ in y direction.

\section{CONCLUSIONS}


The critical factors for the ion source are good plasma uniformity, low operation pressure. The experimental data has suggested using an internal antenna for the ion source because of its capability to operate at lower pressure and produce good uniform plasma density. An external antenna also has the potential problem of back-streaming electrons damaging the rear dielectric window (unless protected by shielding). Although the internal antenna source may be less effective in plasma production both sources can deliver the required current densities $150-250 \mathrm{~mA} / \mathrm{cm}^{2}$ with $<15 \mathrm{~kW}$ of RF- power.

Based on computer simulation, this three-grid extraction system with $6 \sim 8 \mathrm{~cm}$ long slits is expected to deliver the neutral $\mathrm{H}_{1}{ }^{0}$ particle flux of $1.9 \cdot 10^{9}-2.3 \cdot 10^{9}$ particles/s (assuming $90 \% \mathrm{H}_{1}{ }^{+}$fraction and $70 \%$ neutralization) to the target with a full angular divergence of $\sim 40-50 \mathrm{mrad}$ and a beam size $\sim 10 \mathrm{~cm}$ in the y direction.

\section{ACKNOWLEDGMENTS}

This work was supported by NOVA Photonics Inc. and the U.S. Department of Energy under contract number DE-AC02-05CH11231 and DE-FG02-05ER86256.

\section{Figures and figure captions}


Figure 1: Neutral beam injection system block diagram.

Figure 2(Color online): (a) Schematics of the test sources with internal and external antenna. The source is made of aluminum (blue) and has multicusp confinement magnets (red). (b) Photograph of the ion source with internal antenna.

Figure 3(Color online): Extracted ion current vs. operating pressure.

Figure 4(Color online): Extracted ion current vs. RF-power at optimum operation pressures. External antenna is more efficient in producing the plasma but both antennas are capable of producing $150 \mathrm{~mA} / \mathrm{cm}^{2}$ with $<15 \mathrm{~kW}$ of RF-power.

Figure 5(Color online): Plasma density profiles obtained by a movable Langmuir probe $\sim 0.5 \mathrm{~cm}$ from front flange.

Figure 6(Color online): Ion trajectory plot from IBsimu for multiple slit extraction.

Figure 7(Color online): Beam profile in $y$ and $y^{\prime}$ at the target, at $2.5 \mathrm{~m}$ downstream from the last extraction electrode.

\section{REFERENCE}

1 R J Fonck, R J Goldston, R. Kaita and D E Post, Appl. Phys. Lett. 42, 239 (1983). 
2 R C Isler and L E Murray, Appl. Phys. Lett. 42, 355 (1983).

3 R J Fonck, D S Darrow, and K P Jaehnig, Phys. Rev. A 29, 3288 (1984).

4 R J Fonck, P A Duperrex, and S F Paul, Rev. Sci. Instrum. 61, 3487 (1990).

5 F M Levington, R J Fonck, G M Gammel, R Kaita, H W Kugel, E T Powell, and D W

Roberts, Phys. Rev. Lett. 63, 2060 (1989).

${ }^{6}$ J Staples and T Schenkel, Particle Accelerator Confernce, Chigaco, 2108, (2001).

7 J Reijonen, T P Lou, B Tolmachoff, and K N Leung, SPIE, San Diego, 80, (2001).

8 J H Vainionpaa, T Kalvas, S K Hahto and J Reijonen, Rev. Sci. Instrum. 78, 063503 (2007).

9 L T Perkins, G J De Vries, P R Herz, W B Kunkel, K N Leung, D S Pickard, A $\wedge$ Wengrow, and M D Williams, Rev. Sci. Instrum. 67, 1057 (1996).

${ }^{10}$ T. V. Kalvas, Ion Beam simulator, Aug 2007 http://www.cc.jyu.fi/ tvkalvas/code/ibsimu/index.html.

${ }^{11}$ J. E. Boers, PBGUNS version 5.04 Thunderbird Simulations, 2001, http://www.thunderbirdsimulations.com. 
Fig. 7

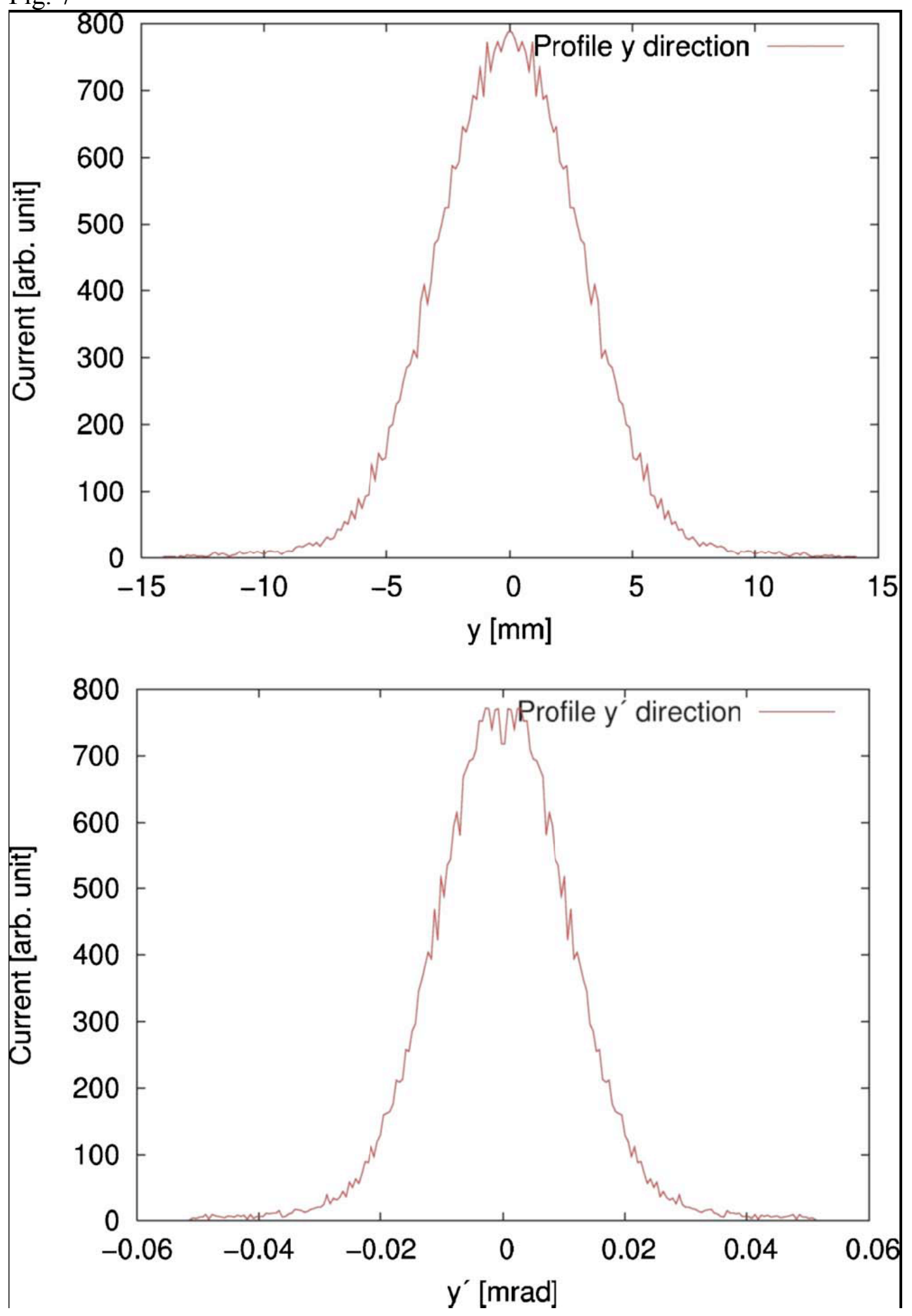


Target plasma

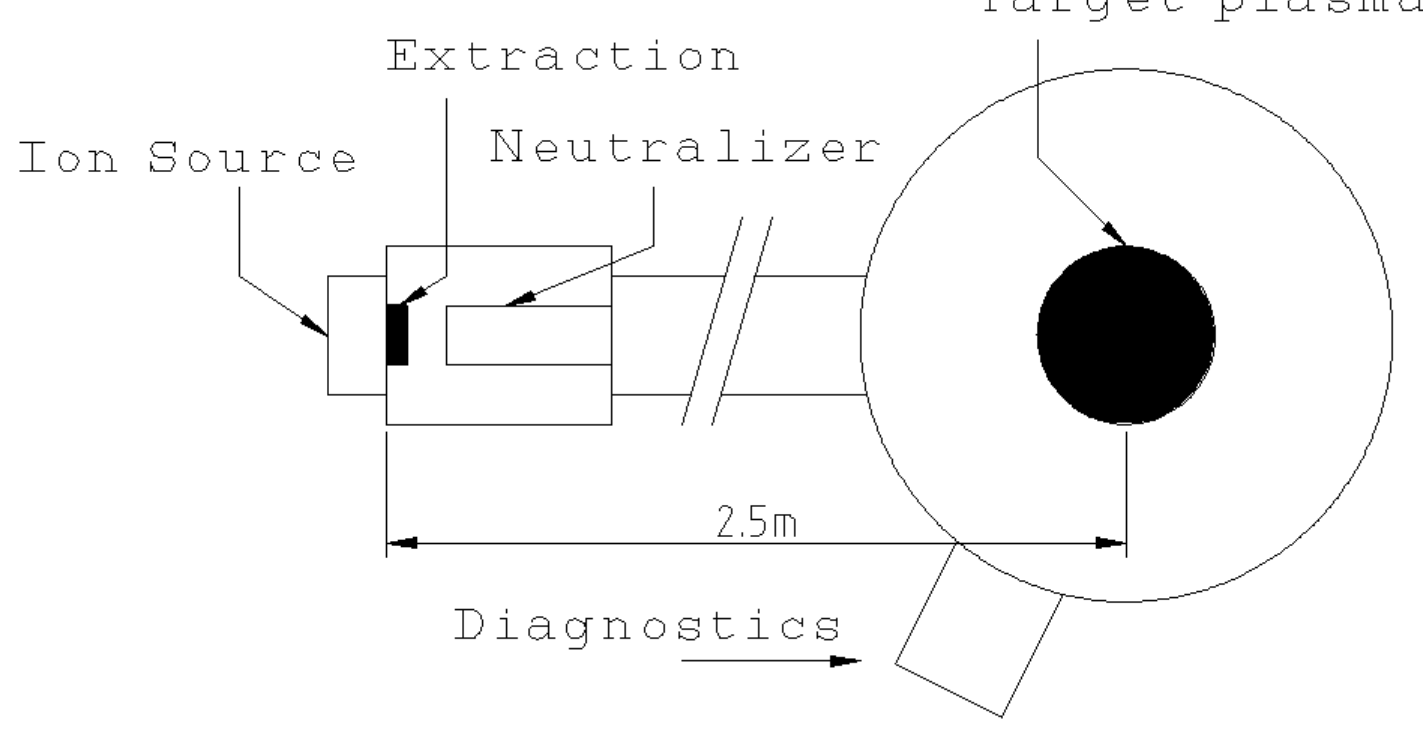

Fig. 1 


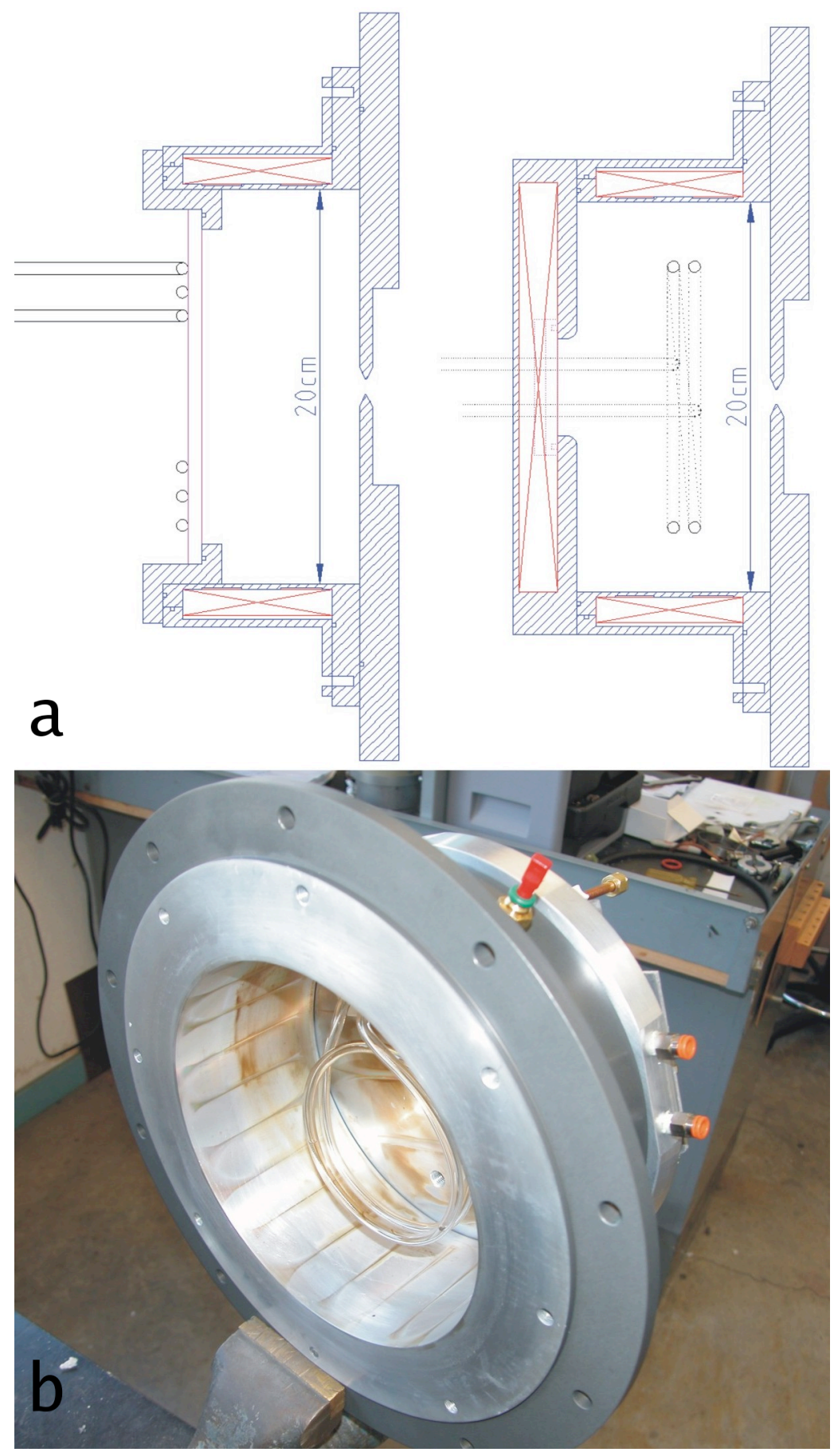

Figures 2, A and B 
Fig 3

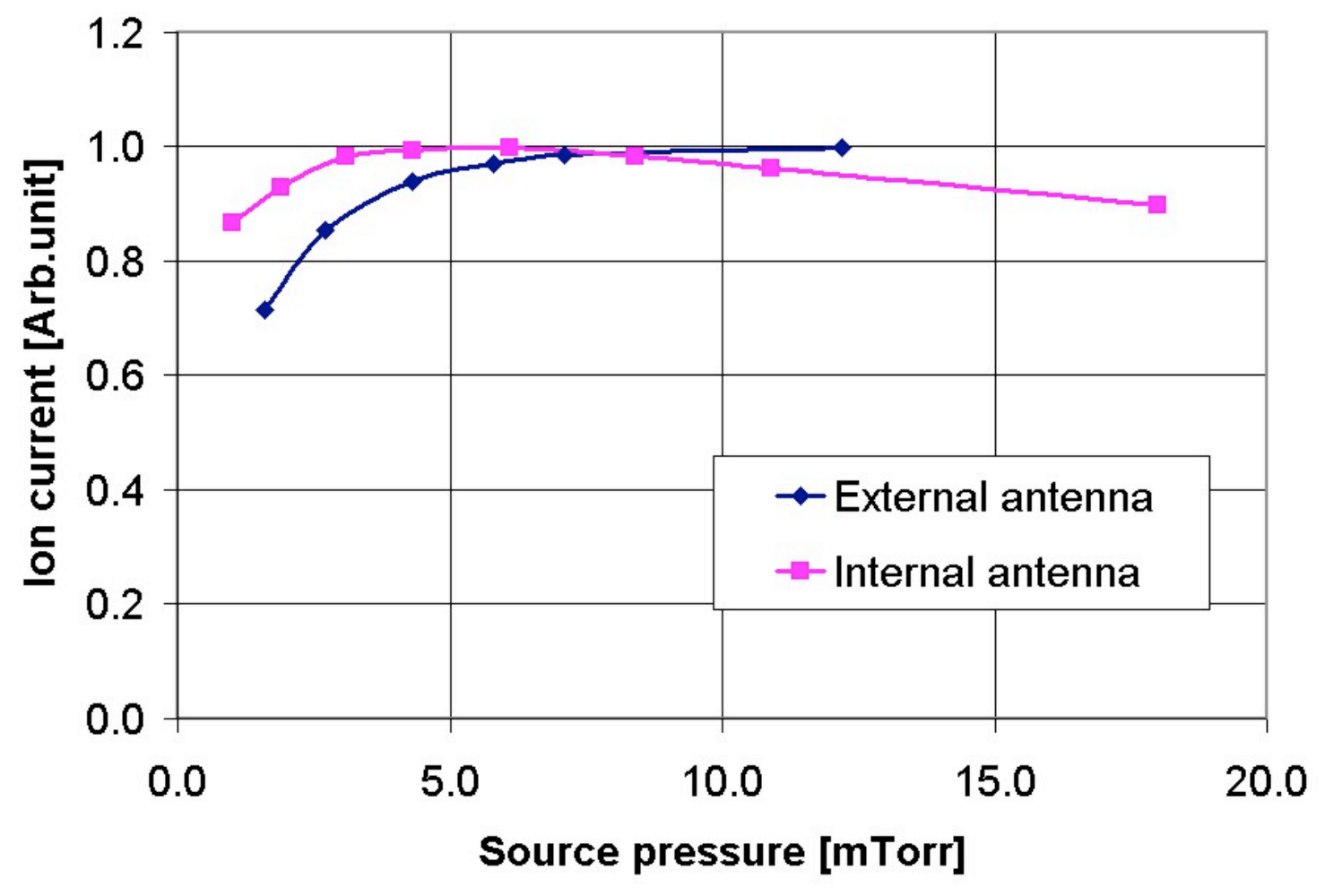


Fig. 4

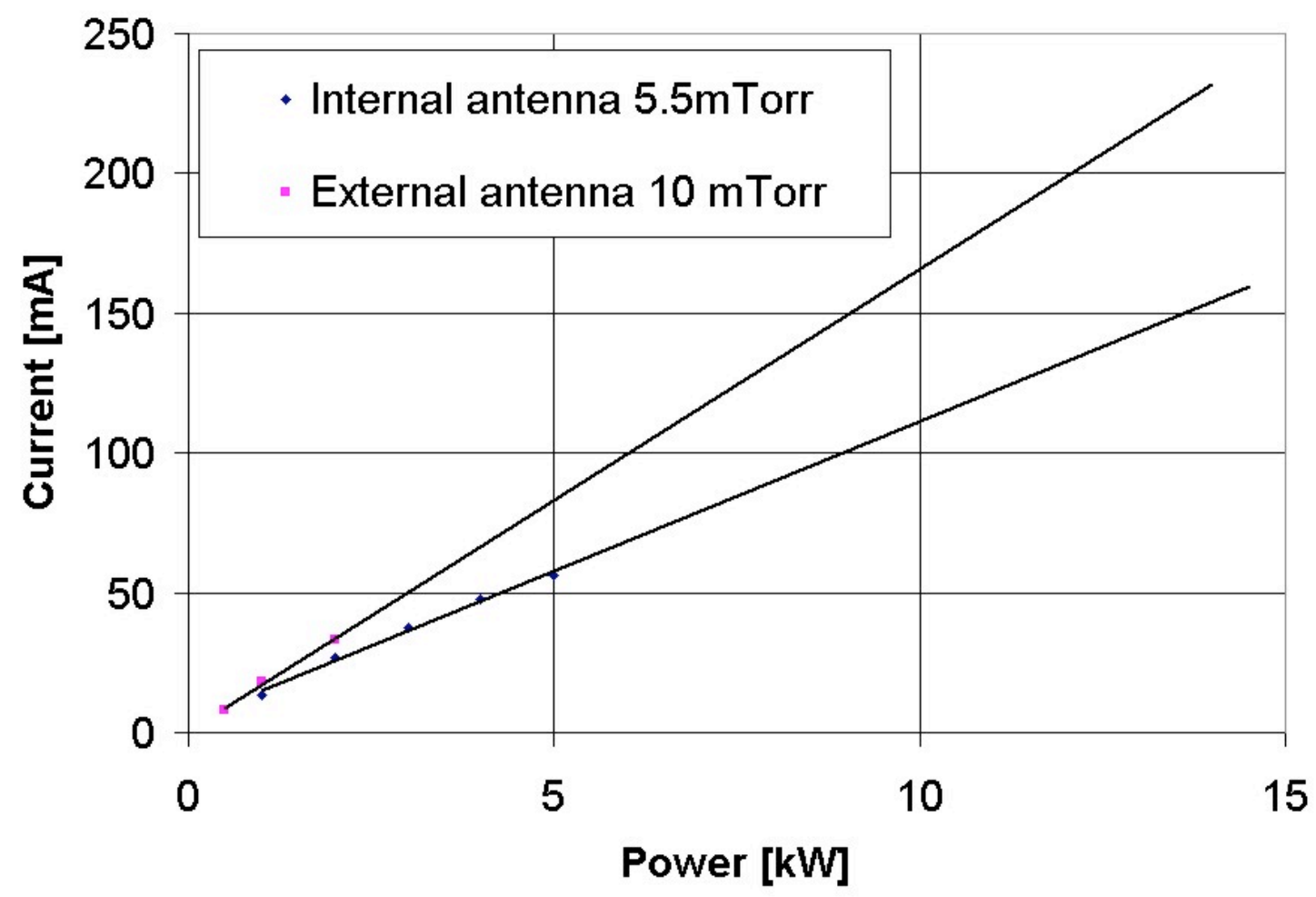


Fig 5

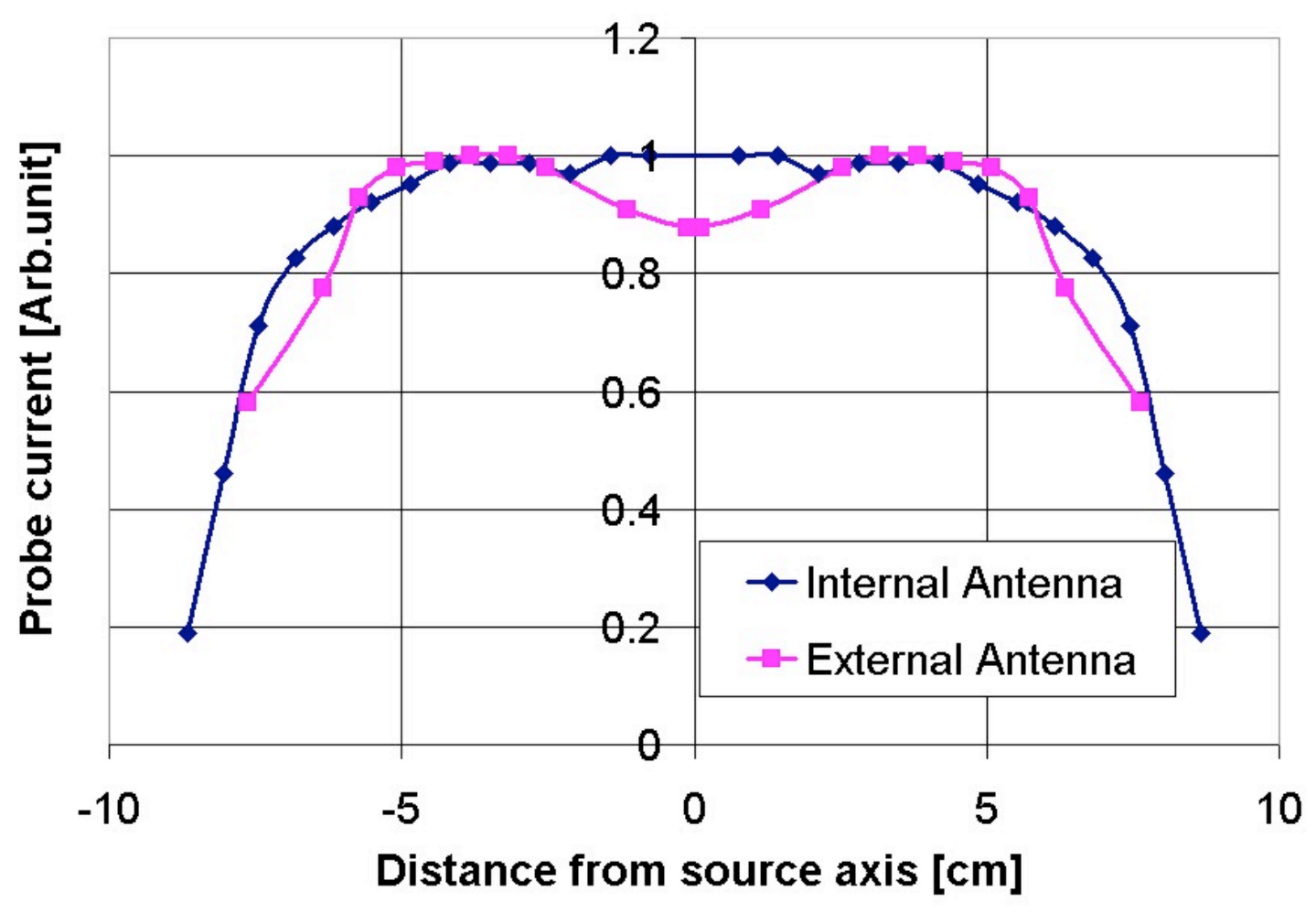


Fig. 6

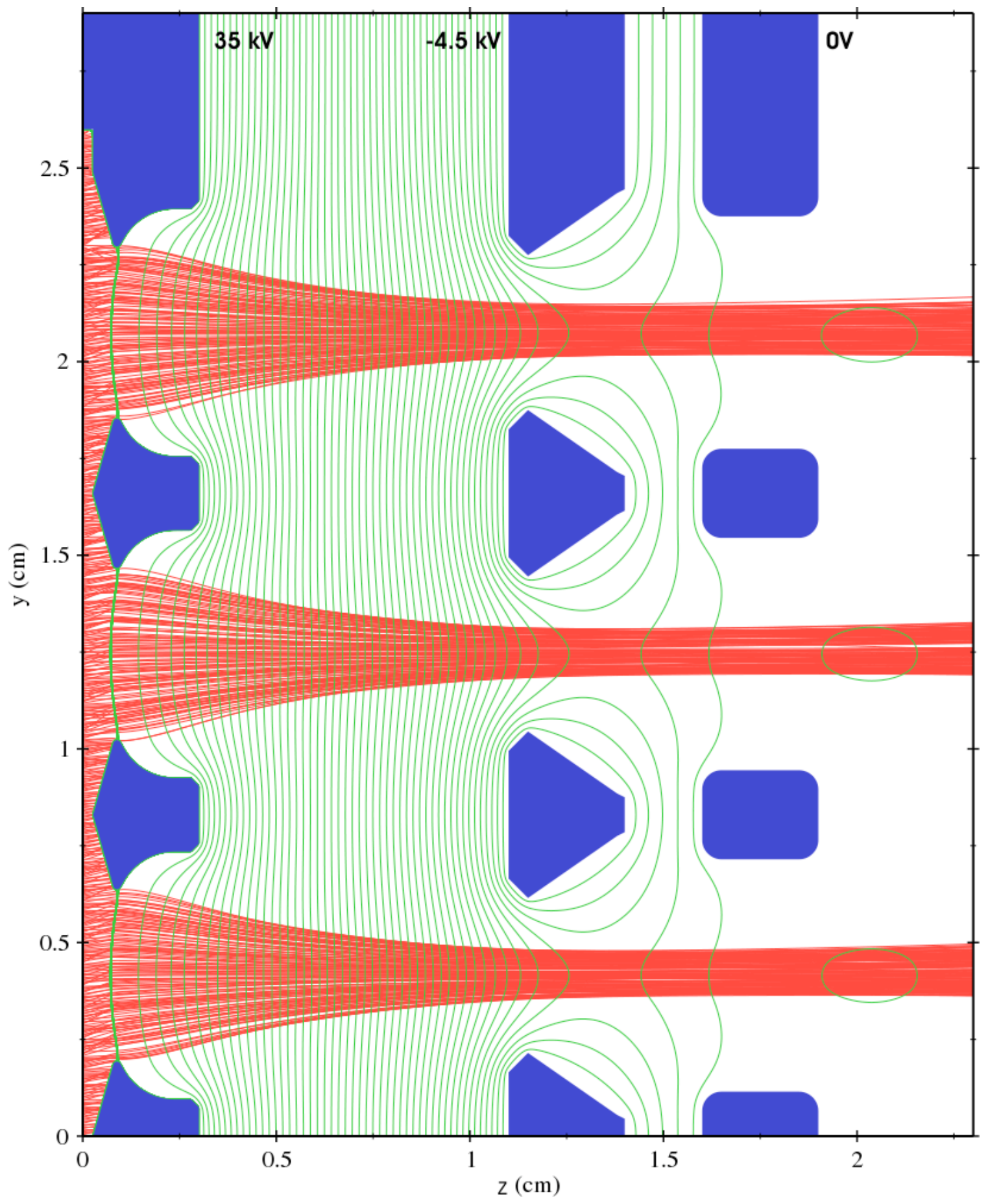

